\title{
Eventos de Chuva Característicos: metodologia adaptada e aplicada a poços de infiltração na cidade de São Carlos-SP
}

\author{
Characteristic Rain Events: adapted methodology applied \\ to infiltration wells São Carlos-SP
}

Luan Serafim Mendes Gonçalves ${ }^{1}$ (D), Bernardo Arantes do Nascimento Teixeira ${ }^{1}$ (C)

${ }^{1}$ Universidade Federal de São Carlos - UFSCar, São Carlos, SP, Brasil. E-mails: luansmg@hotmail.com, bernardo@ufscar.br

Como citar: Gonçalves, L. S. M., \& Teixeira, B. A. N. (2021). Eventos de Chuva Característicos: metodologia adaptada e aplicada a poços de infiltração na cidade de São Carlos-SP. Revista de Gestão de Água da América Latina, 18, e24. https://doi.org/10.21168/rega.v18e24

RESUMO: Frequentemente as cidades brasileiras sofrem com inundações, deslizamentos e erosões causadas pelo escoamento superficial da água da chuva. Os sistemas de drenagem urbanos sustentáveis incorporam técnicas de infiltração, evapotranspiração e retenção do escoamento superficial com o objetivo de mimetizar o ciclo hidrológico pré-urbanização. Este trabalho adapta e aplica a metodologia de eventos de chuva característicos (ECC) a partir de uma série histórica de dados pluviométricos para simulação do comportamento hidrológico de poços de infiltração no município de São Carlos/SP. O método ECC busca auxiliar os profissionais que trabalham com dispositivos de drenagem a entender melhor o comportamento desses em um cenário de chuva real. Foram determinados os ECC para seis intervalos de acordo com percentis da amostra, utilizando os dados da estação automática do Instituto Nacional de Meteorologia (INMET) através de rotina de cálculos programados em Visual Basic for Applications (VBA). Os ECC são obtidos através de métodos estatísticos que permitem a seleção de eventos pluviométricos históricos representativos que podem ser aplicados na simulação do comportamento de dispositivos de drenagem urbana mais sustentável.

Palavras-chave: Eventos de Chuva; Drenagem Urbana; Visual Basic for Applications.

ABSTRACT: Brazilian cities often suffer from floods, landslides and erosion caused by stormwater runoff. Sustainable urban drainage systems incorporate techniques of infiltration, evapotranspiration and retention of the surface runoff in order to mimic the pre-urbanization hydrological cycle. This work adapts and applies the characteristic rain event (CRE) methodology from a historical series of rainfall data to simulate the hydrological behavior of infiltration wells in the municipality of São Carlos/SP. The ECC method aims to help professionals who work with drainage techniques to simulate their behavior in a real rain scenario. CRE were determined for six class intervals according to sample percentiles, using data from the automatic station of the National Institute of Meteorology (INMET) through process programmed in Visual Basic for Applications (VBA). CRE are a mathematically reliable way of choosing a historical rainfall event to simulate the behavior of sustainable urban drainage techniques.

Keywords: Rain Events; Urban Drainage; Visual Basic for Applications.

\section{INTRODUÇÃO}

A partir de meados do século XX o Brasil iniciou intenso processo de urbanização, evoluindo de uma população predominantemente rural (Instituto Brasileiro de Geografia e Estatística, 2001) para estimativas recentes de que há cerca de $85 \%$ da população vivendo em áreas urbanas (Instituto Brasileiro de Geografia e Estatística, 2018). Projeções da ONU (United Nations, 2018) indicam que até 2050 haverá $93 \%$ da população brasileira vivendo em cidades. A fim de reduzir os custos com infraestrutura, os planejadores urbanos têm incentivado um modelo de grandes densidades habitacionais. Todavia, essa filosofia tende a produzir efeitos indesejáveis no manejo das águas

Recebido: Julho 23, 2021. Revisado: Setembro 01, 2021. Aceito: Outubro 28, 2021. 
pluviais urbanas, com aumento das áreas impermeáveis e consequente aumento das vazões de drenagem, sobrecarregando esse sistema (Tucci, 2003).

Um conceito fundamental da hidrologia é o ciclo da água. Os efeitos combinados de urbanização, industrialização e crescimento da população afetam as respostas hidrológicas das bacias. Apesar dos princípios fundamentais serem os mesmos, o ciclo hidrológico é severamente modificado em áreas urbanas (Marsalek et al., 2008). Nas condições pré-urbanização, existe um balanço natural entre precipitação, evapotranspiração, infiltração e escoamento superficial da água da chuva. No entanto, a urbanização reduz a permeabilidade do solo e remove a vegetação natural que intercepta, reduz a velocidade de escoamento superficial e promove a infiltração da água pluvial, aumentando, portanto, o escoamento superficial e modificando o ciclo natural da água (Ballard et al., 2015).

Até meados do século 18, era aceitável o risco de inundações urbanas, sendo considerado um preço a se pagar pelo desenvolvimento próximo à água (Brasil, 2007). Já no século 19, tornou-se padrão a canalização das águas pluviais, principalmente devido à relação direta entre saúde pública e construção de sistemas de drenagem (Ashley et al., 2015). 0 sistema tradicional de drenagem urbana seguia o conceito higienista, projetado de forma a permitir a rápida evacuação das águas pluviais do ambiente urbano. Atualmente, a drenagem urbana evoluiu do modelo clássico focado na proteção à saúde humana e redução de enchentes para uma visão mais sustentável, ampla e multidisciplinar, que considera aspectos sanitários, ambientais, sociais, paisagísticos e econômicos.

Assim, a drenagem urbana sustentável busca mimetizar o ciclo hidrológico natural no ambiente urbano através de medidas estruturais e não-estruturais. Estas podem ser regulamentos, leis, normas, planos diretores, políticas de educação ambiental e planejamento urbano. Já as medidas estruturais incluem a construção de dispositivos de drenagem que retém e infiltram a água da chuva (Baptista et al., 2011).

Estruturas hidráulicas são dimensionadas a partir de chuvas de projeto, que são uma representação simplificada do histórico de precipitações, com um determinado risco de ocorrência. Em projetos de drenagem, é usual a utilização do método racional para estimativa de vazões (Bemfica et al., 2000). Já o método da "curva envelope" é muito utilizado para dimensionamento de dispositivos que possuem algum volume de armazenamento. Neste método, a curva de massa afluente do dispositivo é comparada a curva de massa efluente, sendo a maior diferença entre as duas curvas o volume de dimensionamento do dispositivo. Para simplificação, adota-se a curva efluente constante, enquanto a curva afluente é dada pela altura-duração-frequência das chuvas (Silveira \& Goldenfum, 2007).

Outrossim, o potencial de dano de um evento pluviométrico depende não apenas do volume total de chuva, mas também de outras características como a intensidade de pico e a duração total do evento. As propriedades estatísticas dessas características e sua inter-relação são fundamentais para projetos e estudos científicos (Gaál et al., 2014). Segundo Backhaus \& Fryd (2013) é questionável se os projetistas de dispositivos de drenagem possuem conhecimentos específicos da quantidade de água de chuva precipitada em uma dada região. Observa-se comumente dispositivos superdimensionados, que criam verdadeiras crateras no meio urbano. Peroni \& Teixeira (2019) constataram ainda que dispositivos de drenagem compensatória são projetados apenas com função hidrológica, sem integração com seu entorno para funções sociais, esportivas e embelezamento da paisagem.

Diante disso, para ajudar a preencher a lacuna entre projetos hidraulicamente eficientes e que agreguem valores de amenidade para a cidade, a metodologia dos "Eventos de Chuva Característicos (ECC)" proposto por Andersen et al. (2017) pode ser adaptada e empregada em vários cenários, criando projetos de dispositivos de drenagem que transformam a água da chuva em um elemento agradável para a cidade. DiGiovanni (2018) comenta que o método ECC foi desenvolvido com o objetivo de conectar engenharia e urbanismo, de forma a permitir a urbanistas visualizarem como a água vai se apresentar nos dispositivos sustentáveis.

Indubitavelmente a modelagem de dispositivos de drenagem urbana sustentáveis antes da sua instalação ou como uma verificação do projeto as built melhora o desempenho geral por meio da análise de diversos cenários de chuva, permitindo a identificação de consequências de projeto não previstas, ou como uma ferramenta para estudar parâmetros de balanço hídrico, que são difíceis de medir em diferentes condições meteorológicas ou climáticas (Alikhani et al., 2019). Dunkerley (2015) observa que existem muitas abordagens para delinear o que é um evento de chuva, porém normalmente adota-se um intervalo mínimo de tempo para esta tarefa. Para determinar esse período de tempo, pode-se considerar que um evento de chuva deve estar flanqueado por intervalos de tempo sem chuvas maiores que o período de chuva, ou ainda especificar um intervalo de tempo em horas para separar os eventos.

Palynchuk \& Guo (2007) observam que existem algumas formas básicas de determinação da chuva para estudos hidrológicos, sendo que cada técnica tem um uso adequado, sendo elas: Séries 
Contínuas, onde todos os registros históricos da distribuição da chuva são utilizados em modelagens contínuas; Eventos de Chuva Especiais, para análises de grandes eventos, como no caso de furacões; Chuvas de Projeto IDF, onde os valores da relação intensidade-duração-frequência (IDF) são aplicados na determinação de hietogramas de projeto; e por fim os Eventos Pluviométricos, onde as chuvas são agregadas em eventos com um certo tempo entre eventos. Comparando o método dos eventos pluviométricos com o das chuvas de projeto IDF, o método dos eventos é mais realístico, já que considera a duração e volume real de eventos de chuva enquanto que a curva IDF não mede explicitamente o evento, assumindo uma concordância de tempo e intensidade da chuva, o que não e justificável em todos os casos.

Por fim, o método ECC foi originalmente desenvolvido pois os projetos de dispositivos de drenagem pluvial são dimensionados para os piores cenários, usando eventos de chuva sintéticos representando o pior caso imaginável com base em propriedades estatísticas da chuva com um certo risco de ocorrência. Essa filosofia de blindagem contra falhas é normalmente utilizada em projetos de infraestrutura. Esses eventos de chuva sintéticos determinam o volume geral de detenção-retenção exigido de um dispositivo. Dentro deste requisito, a forma do dispositivo pode variar, assim como pode ser feita a associação de diversos dispositivos, e é neste ponto que as habilidades de projeto do urbanista são necessárias. Para estimar como a água aparecerá no dispositivo e o balanço hídrico do mesmo, é útil obter mais informações sobre as chuvas. É possível usar os métodos estatísticos clássicos ou criar chuvas sintéticas, mas escolhendo alguns eventos históricos representativos da chuva haverá uma melhor previsão do funcionamento das estruturas de drenagem em eventos semelhantes (Andersen et al., 2017).

Mediante o exposto, este trabalho teve como objetivos adaptar a metodologia de Eventos de Chuva Característicos proposta por Andersen et al. (2017), desenvolver uma rotina de cálculos para extração desses eventos a partir de uma série histórica de dados pluviométricos e aplicar os ECC obtidos em poços de infiltração no munícipio de São Carlos - SP.

\section{METODOLOGIA}

A metodologia utilizada neste trabalho foi separada em cinco fases, descritas a seguir. A fim de aplicar a metodologia adaptada dos ECC foram utilizados dados pluviométricos da cidade de São Carlos (SP) e um poço de infiltração projetado de acordo com as diretrizes municipais. 0 método ECC pode ser dividido em três fases principais: determinação das características dos eventos históricos, determinação de classes de chuva de acordo com percentis da amostra e seleção do evento de chuva característico.

\section{Obtenção dos dados pluviométricos}

Os dados pluviométricos foram cedidos pelo INMET a partir de solicitação no banco de dados meteorológicos (BDMEP) para a estação meteorológica de observação de superfície automática (EMA) de São Carlos/SP. Os dados fornecidos estão agregados em intervalos de duração horária.

A estação meteorológica automática obtém os dados pluviométricos através de contador de pulso e medidos a cada 10 segundos. A EMA de São Carlos está implantada em latitude: 2158'49.02"S e longitude: 4753'2.24"O (DATUM WGS84) nas instalações da Universidade Federal de São Carlos e iniciou a coleta de dados em 05/09/2006 (Instituto Nacional de Meteorologia, 2011). A série pluviométrica utilizada neste trabalho tem início na data de inauguração da estação e vai até 05/05/2021.

\section{Determinação das características de um evento pluviométrico histórico}

Neste estudo o tempo entre eventos foi determinado como sendo $24 \mathrm{~h}$, regra prática normalmente adotada para que um dispositivo de drenagem urbana possa ser esvaziado, estando apto após esse período a receber o próximo evento pluviométrico (Andersen et al., 2017; Oliveira et al., 2016; Beecham \& Chowdhury, 2012). Este tempo para esvaziamento do dispositivo assegura que em regiões tropicais e subtropicais alguns organismos vetores de doenças, como mosquitos e pernilongos, não se desenvolvam (Baptista et al., 2011).

Além disso, a intensidade mínima a ser considerada no evento é de $1 \mathrm{~mm} \mathrm{~h}^{-1}$. Esse valor mínimo representa as perdas iniciais dos sistemas de drenagem. Chuvas com registro abaixo da intensidade $1 \mathrm{~mm} \mathrm{~h}^{-1}$ são consideradas nulas, pois aumentariam a duração do evento sem efetivamente aumentar o volume de escoamento superficial. Caso haja registro de intensidade $1 \mathrm{~mm} \mathrm{~h}^{-1}$ entre registros de intensidades maiores do que $1 \mathrm{~mm} \mathrm{~h}^{-1}$ e dentro do tempo entre eventos de $24 \mathrm{~h}$, esse registro é considerado para a construção do evento. A Figura 1 ilustra as considerações para individualização de eventos de chuva. 


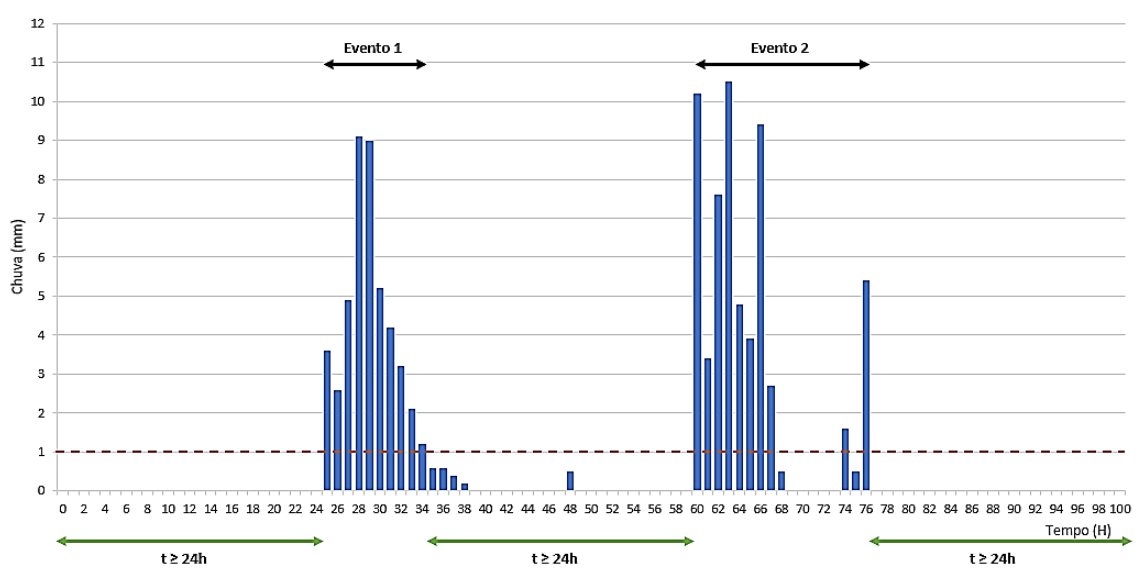

Figura 1 - Eventos de Chuva.

Para consolidação do evento, caso o bloco horário subsequente estiver separado por um período seco de ao menos o intervalo determinado de $24 \mathrm{~h}$, esse bloco é agregado ao evento. Caso o período seco seja maior que o intervalo entre eventos determinado, então considera-se eventos separados. Cada evento é caracterizado por sua intensidade e duração.

\section{Determinação das Classes dos Eventos de Chuva}

Andersen et al. (2017) e Sørup et al. (2016) classificaram em seu trabalho eventos de chuva de acordo com tempos de retorno de valores rotineiros, de projeto e extremos de acordo com a legislação norueguesa e necessidades dos estudos. Khan, Chapa e Hack (2020) por sua vez agruparam eventos de chuva de acordo com o 50ㅜ $75^{\circ}, 90^{\circ}$ e $95^{\circ}$ percentil da amostra. Para este trabalho, foram adotadas

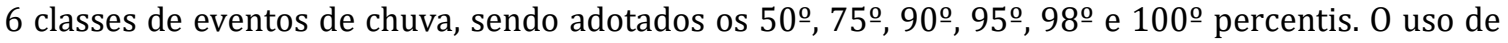
mais classes de chuva neste trabalho é justificado devido as características da amostra, que apresenta grande amplitude dos valores de altura pluviométrica total dos eventos.

Os eventos de chuva foram tabulados em ordem decrescente de acordo com a altura total de chuva do evento, e então calculada a frequência relativa de cada um dos dados. Os eventos de chuva não foram separados por anos hidrológicos ou anos civis, sendo considerado todos os eventos registrados.

\section{Seleção do Evento de Chuva Característico (ECC)}

Para cada uma das seis classes de eventos, que representam os percentis adotados, foi selecionado um ECC. Todos os eventos de chuva históricos foram considerados. Foi calculada a média de chuva para cada hora, produzindo, portanto, um evento de chuva fictício que é uma distribuição média daquela classe. Para encontrar o ECC entre todos os eventos históricos, foi utilizado o método dos mínimos quadrados entre os dados históricos e a chuva fictícia, considerando também a duração do evento, como mostra a Equação 3.

$$
\min (S S Q)=\min \left(\sum_{i=1}^{N} \frac{\left(y_{i}-\ddot{y}_{i}\right)^{2}}{N}\right)
$$

Onde $y_{i}$ é o evento histórico em ordem horária $\mathrm{i}, \ddot{y}_{i}$ é a intensidade da chuva média fictícia em ordem horária e $N$ é o número de horas da classe do evento. 0 evento de chuva histórico que mais se aproxima da chuva fictícia é o que tem a melhor distribuição de alturas de chuva, e será, portanto, o ECC.

Para extração dos eventos pluviométricos a partir dos dados do INMET, foi elaborada uma rotina de cálculo em linguagem Visual Basic for Applications (VBA), que é utilizada em soluções de engenharia por ser reconhecidamente uma linguagem com baixo tempo de programação, compatível com recursos limitados, e de fácil controle de qualidade. A Figura 2 mostra o algoritmo simplificado utilizado para extração dos eventos de chuva históricos (ECH). 


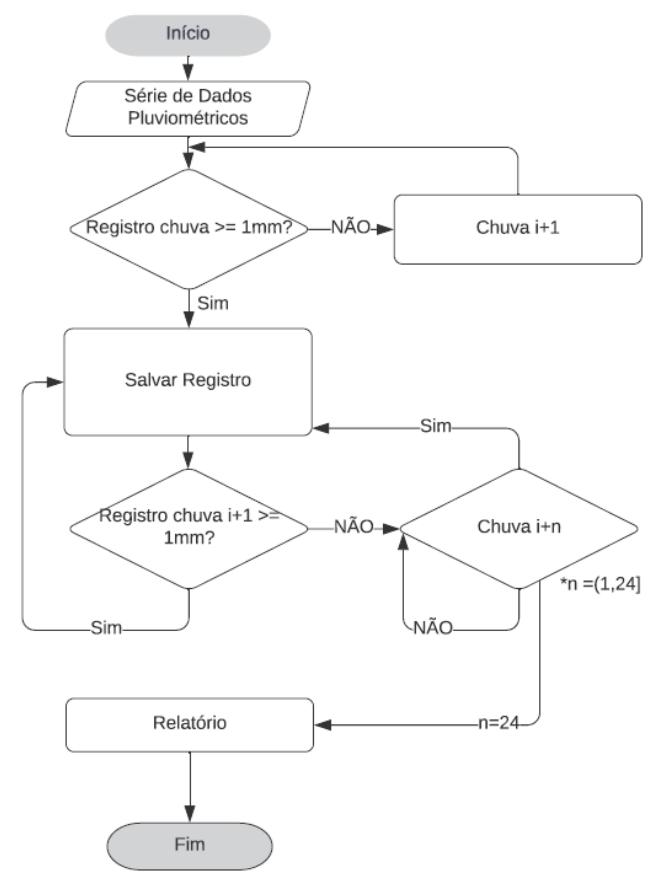

Figura 2 - Algoritmo simplificado usado para extração dos ECH.

\section{Simulação dos ECC em poço de infiltração}

As recomendações de dimensionamento de dispositivos de drenagem são diferentes entre cada jurisdição. 0 Código de Obras e Edificações do município de São Carlos (Lei 15958/2011) por exemplo, determina que seja implantado no lote poço de infiltração cujo volume seja de no mínimo $5 \mathrm{~L} / \mathrm{m}^{2}$ impermeabilizado.

Assim, o poço de infiltração que será simulado está construído no campus da UFSCar para tratamento da água do telhado do Laboratório de Biopolímeros, que possui duas águas, sendo que uma delas é associada a pátio pavimentado no térreo com área total de contribuição de $287,5 \mathrm{~m}^{2}$, conforme ilustrado na Figura 3.0 poço foi construído com anéis de concreto de diâmetro interno de $110 \mathrm{~cm}$ e altura útil até $\mathrm{o}$ extravasor de 200 $\mathrm{cm}$, não havendo material de enchimento e sendo permitido a infiltração pelo fundo e laterais do poço. 0 solo do local pode ser classificado de acordo nomenclatura USDA como sendo Franco Argilo Arenoso. Ferreira (2016) determinou o coeficiente de permeabilidade saturado do solo local pelo método de Pratt e Powel como sendo de $17,3 \mathrm{~mm} \mathrm{~h}^{-1}$.

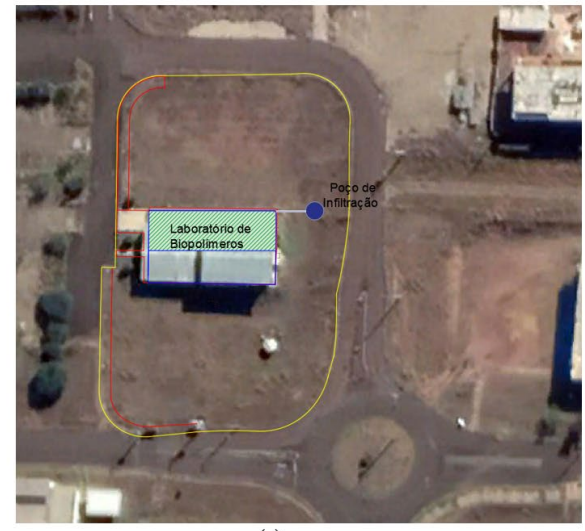

(a)

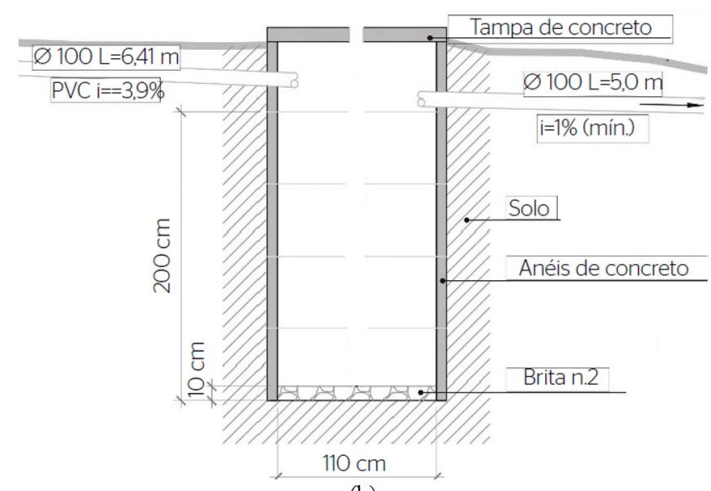

(b)

Figura 3 - Área de estudo. a) detalhe da localização do laboratório de biopolímeros e da EMA; b) detalhe do poço de infiltração.

Para cálculo da taxa de infiltração do poço foi utilizado o método Green-Ampt modificado por Mein e Larson. Esse modelo é de grande precisão e necessita como parâmetros de entrada: 
condutividade hidráulica, déficit de saturação do solo, porosidade e a espessura da zona capilar do solo. A exceção da condutividade hidráulica que foi determinada em laboratório, os outros parâmetros do solo foram definidos de acordo com o trabalho de Rawls et al. (1983). 0 método Green-Ampt pode ser descrito pela Equação 4 para o cálculo da taxa de infiltração.

$$
f(t)=K\left(\frac{\Psi * \Delta \theta}{F(t)}+1\right)
$$

Onde: $f(t)$ é a taxa de infiltração no tempo $t ; K$ é a condutividade hidráulica, $\Psi$ é a sucção na frente de molhamento, $\Delta \theta$ é o acréscimo de umidade no solo pela infiltração. A Equação 5 permite calcular o $\Delta \theta$ através da saturação e porosidade.

$$
\Delta \theta=\left(1-S_{e}\right) * \theta_{e}
$$

Onde: $S_{e}$ é a saturação efetiva e $\theta_{e}$ é a porosidade efetiva

A Equação 4 nos mostra que a capacidade de infiltração depende do volume infiltrado, que por sua vez depende da taxa de infiltração em cada intervalo de intervalo. A fim de evitar erros numéricos em intervalos de tempo maiores, a Equação 6 é uma forma integrada mais adequada para o cálculo da infiltração. Substituindo $f(t)$ por $\partial F / \partial t$, temos:

$$
F(t)=K t+\Psi^{*} \Delta \theta^{*} \ln \left(1+\frac{F(t)}{\Psi^{*} \Delta \theta}\right)
$$

Onde $F(t)$ é a infiltração acumulada. Inicialmente $F(t)$ é estimada, e a partir de iterações de convergência é encontrado o $F(t)$ real para cada intervalo de tempo. A capacidade de evaporação do poço foi desprezada nesta modelagem, por ser dispositivo pontual fechado.

O princípio de conservação de massa e o método não linear de reservação são utilizados para cálculo do balanço hídrico do poço de infiltração (Equação 7).

$\frac{\partial p}{\partial t}=i+q 0-e-f-q 1$

Sendo $p$ a profundidade de água no poço, $i$ é a altura de chuva no local, $q 0$ são os escoamentos superficiais a montante, e é a taxa de evaporação, $f$ é a taxa de infiltração no solo, $q 1$ é a taxa de extravasão e $t$ é o tempo. Através de programação em VBA e utilizando as Equações 6 e 7 foi possível calcular o balanço hídrico para cada evento, assim como a evolução da infiltração e reservação do poço ao longo do tempo.

\section{RESULTADOS}

\section{Categorização dos Eventos}

Foram extraídos um total de 770 eventos na série analisada e então classificados em 6 classes

\begin{tabular}{|c|c|c|c|c|}
\hline Classe & Descrição das categorias & $\begin{array}{l}\text { Qtde de } \\
\text { Eventos }\end{array}$ & $\%$ de Eventos & $\begin{array}{c}\text { Intervalos de } \\
\text { chuva (mm) }\end{array}$ \\
\hline $\mathrm{I}$ & Chuvas até o $50^{\circ}$ percentil & 385 & 50 & $1,0-13,2$ \\
\hline II & Chuvas entre o $50^{\circ}$ percentil e o $75^{\circ}$ percentil & 193 & 25 & $13,2-30,0$ \\
\hline III & Chuvas entre o $75^{\circ}$ percentil e o $90^{\circ}$ percentil & 115 & 15 & $30,0-61,0$ \\
\hline IV & Chuvas entre o $90^{\circ}$ percentil e o $95^{\circ}$ percentil & 39 & 5 & $61,0-88,6$ \\
\hline $\mathrm{V}$ & Chuvas entre o $95^{\circ}$ percentil e o $98^{\circ}$ percentil & 23 & 3 & $88,6-121,8$ \\
\hline VI & Chuva acima do $98^{\circ}$ percentil & 15 & 2 & $121,8-310,0$ \\
\hline
\end{tabular}
conforme descritas na Tabela 1

Tabela 1- Categorias de chuva do método ECC para São Carlos. 
A Figura 4 foi construída a partir da altura total de chuva em $\mathrm{mm}$ de cada evento em relação a sua duração total. Cada ponto na figura representa um único evento de chuva, totalizando os 770 eventos. As classes de acordo com os percentis estão representadas pela altura de chuva. Esta figura mostra que existe uma certa relação de proporcionalidade entre duração do evento e volume total precipitado. A grande maioria dos eventos possuem duração menor do que $12 \mathrm{~h}$. É possível notar ainda um evento extremo registrado com $310 \mathrm{~mm}$ de chuva, que apresenta grande disparidade quanto à sua altura total precipitada e duração em relação aos demais eventos.

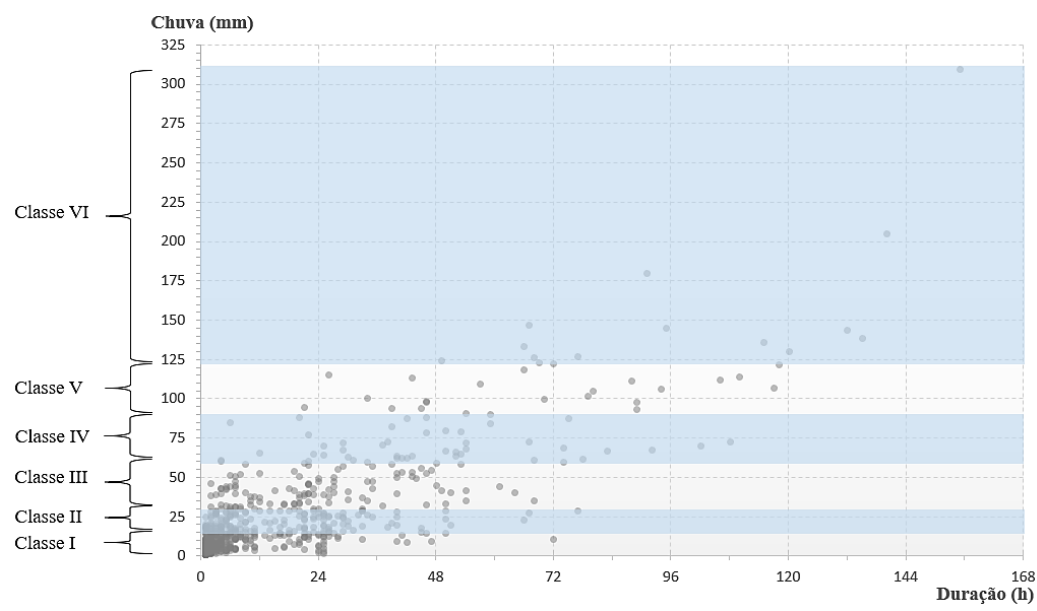

Figura 4 - Altura dos eventos de chuva vs. Duração dos eventos de chuva.

Na Figura 5 estão plotadas as alturas dos eventos pluviométricos classificados de acordo com a proporção da população de dados. A linha de tendência exponencial dos dados mostra excelente ajuste para as duas primeiras classes, com aumento da discrepância a partir da terceira classe. 0 coeficiente de determinação foi calculado como sendo 0,99, o que mostra bom ajuste à amostra. Observa-se ainda nessa figura, que os eventos com volume total precipitado de até $50 \mathrm{~mm}$ representam cerca de $85 \%$ dos eventos de chuva registrados, enquanto que os eventos com mais de $100 \mathrm{~mm}$ ocorrem em apenas $4 \%$ dos registros históricos de chuva.

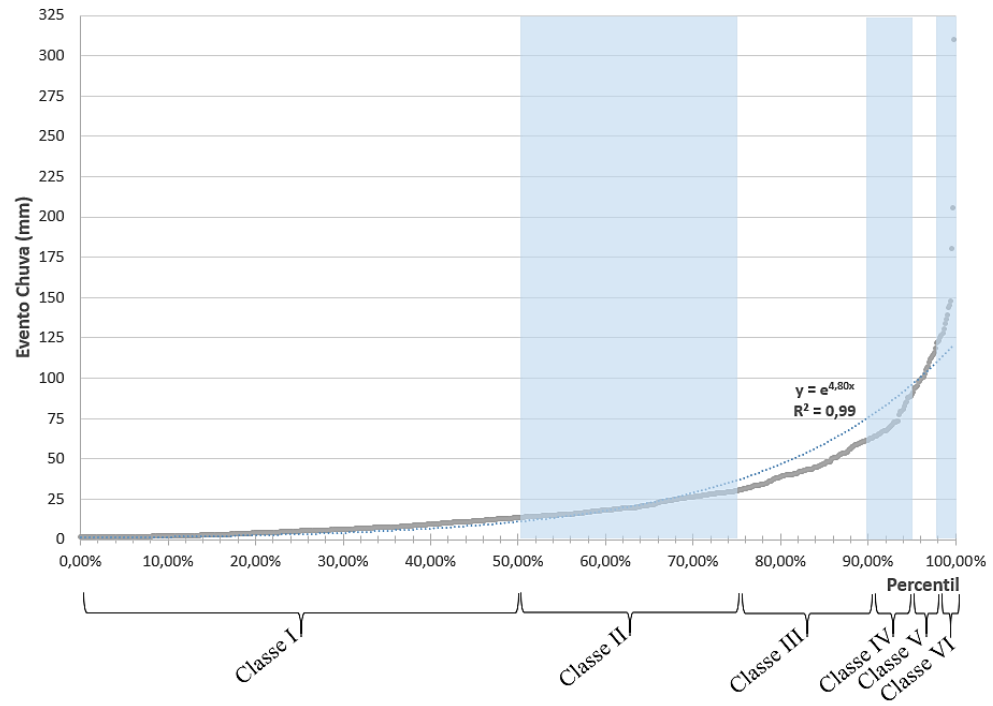

Figura 5 - Eventos de chuva (mm) vs. proporção da população.

\section{Seleção dos Eventos de Chuva Característicos (ECC)}

A Figura 6 mostra as seis classes de eventos determinadas de acordo com os percentis dos eventos de chuva históricos, construída de acordo com a intensidade pluviométrica do evento em $\mathrm{mm} \mathrm{h}^{-1}$ pela duração total do mesmo em horas. As escalas foram ajustadas para melhor visualização dos dados de cada classe. 
Todos os eventos históricos de uma categoria são mostrados em linhas cinzas. A linha laranja representa um evento sintético médio daquela categoria, e a linha vermelha representa o evento histórico que mais se aproxima do evento médio obtido pelo método dos mínimos quadrados, sendo este o evento de chuva característico daquela categoria. São apresentados ainda em cada categoria o número de eventos (NoE), a duração média dos eventos $(\mu)$, e o desvio padrão da duração dos eventos $(\sigma)$.

De forma geral, há grande diferença nas durações dos eventos em cada classe, sendo que para a primeira classe a duração média dos eventos é de 4,4 horas, e para a sexta classe obtivemos eventos de duração média de 96,7 horas, pouco mais do que quatro dias. Apesar do desvio padrão da duração dos eventos ser elevado, o que indica a distribuição dos dados por uma ampla gama de valores, a classificação dos dados por percentis de acordo com o volume total da chuva também refletiu em uma boa classificação para a duração dos eventos. A Figura 6 nos mostra ainda o comportamento de um evento de chuva real, sendo em sua maioria caraterizados por vários picos de chuva associados a períodos secos. Na classe cinco é possível identificar o evento com maior intensidade horária registrado, com 73,8 mm.
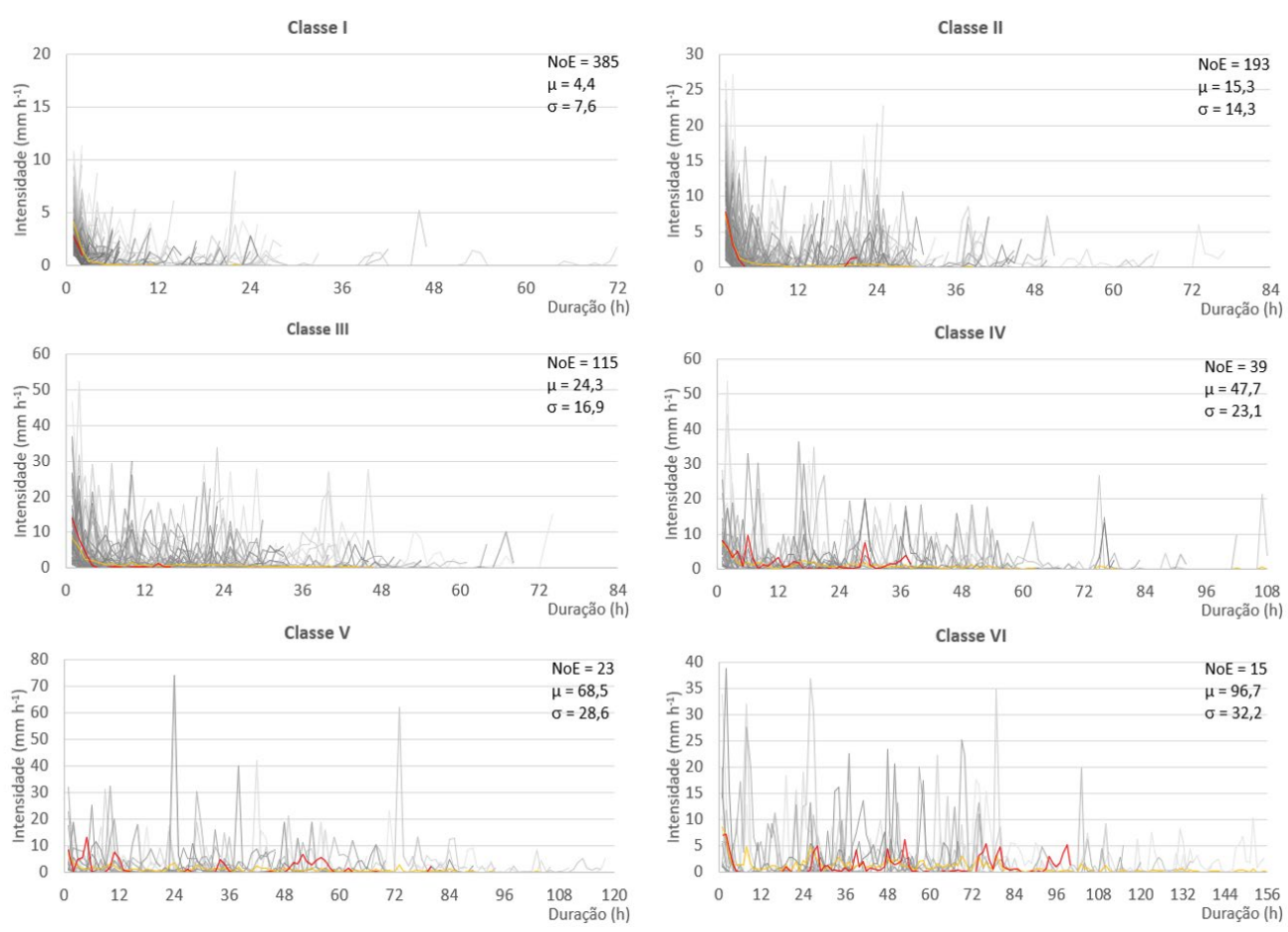

Figura 6 - Determinação dos Eventos de Chuva Característicos.

As propriedades dos Eventos de Chuva Característicos selecionados estão descritas na Tabela 2. Os ECC foram numerados de 1 a 6 de acordo com sua classe. Algumas particularidades podem ser notadas nesta tabela, como o fato do ECC 5, que possui uma duração total de 80 horas, apresentar 41 horas de registros nulos, havendo, portanto, muitos períodos curtos de chuva associados a períodos curtos secos. Observa-se também que o ECC 6, que possui o maior volume total, apresentou intensidades máximas e médias pequenas comparado aos demais. 0 ECC 1, que é uma chuva de menor volume total e duração, apresentou a maior intensidade média dos eventos característicos. Apenas o ECC 2 apresentou intensidade média abaixo de $1 \mathrm{~mm} \mathrm{~h}^{-1}$.

Tabela 2 - Propriedades dos Eventos de Chuva Característicos.

\begin{tabular}{|c|c|c|c|c|c|c|}
\hline ECC & $\begin{array}{l}\text { Chuva } \\
(\mathrm{mm})\end{array}$ & $\begin{array}{l}\text { Duração } \\
\text { (horas) }\end{array}$ & $\begin{array}{c}\text { Horas com } \\
\text { intensidade nula }\end{array}$ & $\begin{array}{c}\text { Imín } \\
\left(\mathrm{mm} \mathrm{h}^{-1}\right)\end{array}$ & $\begin{array}{c}\text { Imáx } \\
\left(\mathrm{mm} \mathrm{h}^{-1}\right)\end{array}$ & $\begin{array}{c}\text { IMéd } \\
\left(\mathrm{mm} \mathrm{h}^{-1}\right)\end{array}$ \\
\hline 1 & 5,6 & 2 & 0 & 1,8 & 3,8 & 2,8 \\
\hline 2 & 14,6 & 21 & 16 & 1,0 & 7,8 & 0,7 \\
\hline 3 & 40 & 22 & 5 & 0,2 & 14 & 1,8 \\
\hline 4 & 73,2 & 38 & 5 & 0,2 & 9,6 & 1,9 \\
\hline 5 & 105 & 80 & 41 & 0,2 & 13,2 & 1,3 \\
\hline 6 & 138,8 & 135 & 50 & 0,2 & 7,2 & 1,0 \\
\hline
\end{tabular}




\section{Simulação do poço de infiltração}

0 poço de infiltração teve seu comportamento simulado para cada um dos ECC. A Figura 7 mostra o resumo agregado do balanço hídrico e a Figura 8 traz a evolução do comportamento hidrológico do poço ao longo do tempo.

0 poço em estudo teve bom desempenho quanto a infiltração do escoamento superficial recebido das áreas impermeabilizadas. Os volumes de escoamento superficial gerados pelos ECC 1 e 2 foram totalmente infiltrados pelo poço, o que nos indica que o poço terá desempenho excelente até o $75^{\circ}$ percentil dos eventos de chuva. Mesmo nos eventos em que ocorreram extravasão do poço, houve grande contribuição para redução do escoamento superficial.

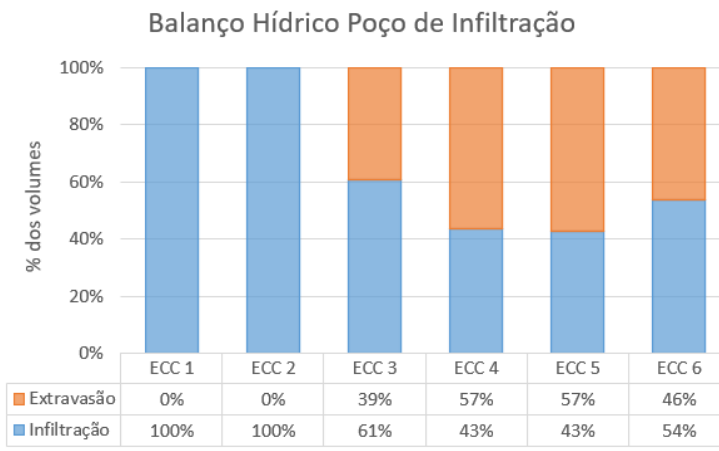

Figura 7 - Balanço Hídrico para os ECC.

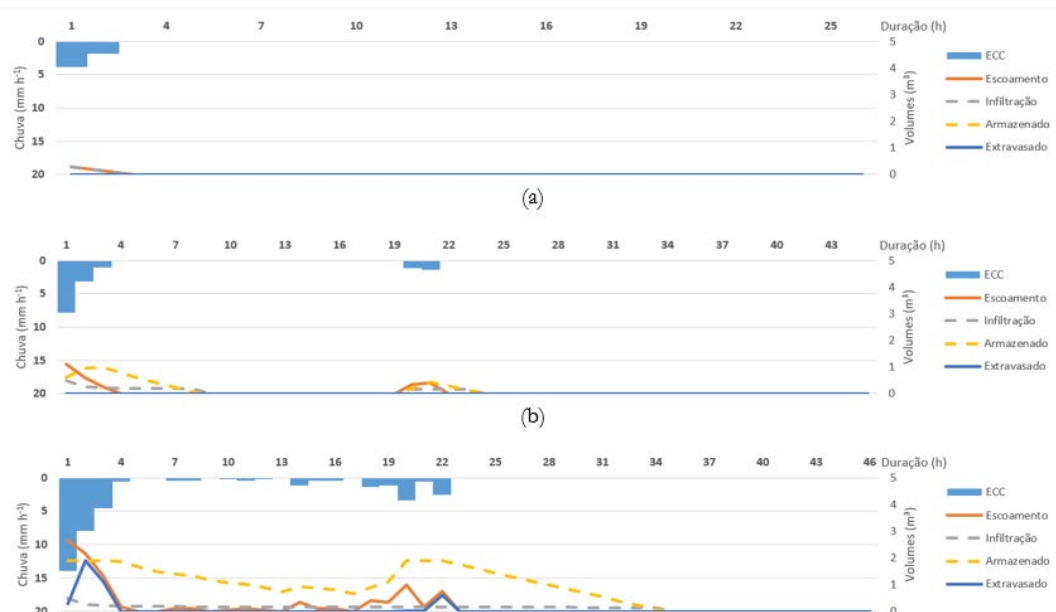

(c)

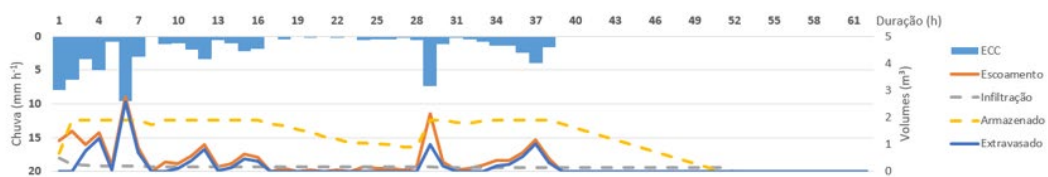

(d)

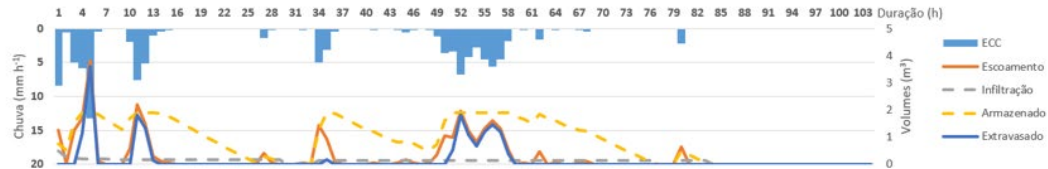

(e)

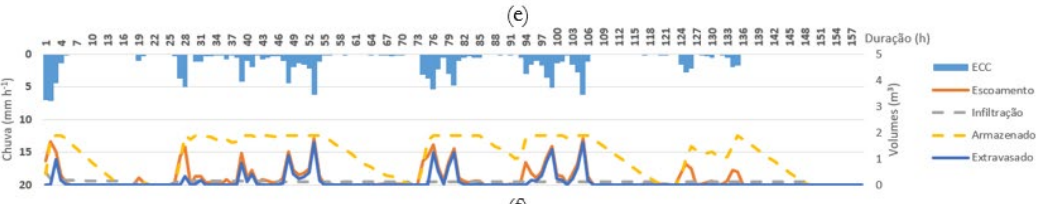

(f)

Figura 8 - evolução do comportamento hidrológico do poço de infiltração isolado. a) ECC 1; b) ECC 2; c) ECC 3; d) ECC 4; e) ECC 5; f) ECC 6 
Através da Figura 8 é possível ainda visualizar a importância no uso de eventos de chuva reais para simulação de dispositivos de drenagem sustentável que trabalham majoritariamente via reservação com posterior infiltração para controle das águas de escoamento. Para os ECC 3 e 4 o poço de infiltração não foi capaz de infiltrar a água armazenada nos períodos secos antes da chegada de um novo pico de chuva, o que resultou em maiores volume de extravasão pontual. Já para os eventos 5 e 6, o poço conseguiu infiltrar todo o volume armazenado antes da chegada de um novo pico de chuva, atenuando a extravasão pontual. Apesar de características distintas entre os eventos de chuva característicos, o desempenho final do poço quando sujeito aos ECC 4 e 5 foi exatamente o mesmo. No caso do ECC 6, que representa os maiores volumes totais de chuva, o poço obteve um desempenho ligeiramente superior em relação aos ECC 4 e 5, devido principalmente aos períodos secos com intervalo suficiente para permitir o completo esvaziamento do poço.

De forma geral, a capacidade de armazenamento do poço é suficiente para suportar pequenos picos de escoamento superficial, possibilitando a infiltração nos intervalos de tempo seguintes até o 75ํㅜ percentil dos eventos de chuva. Como possíveis soluções para melhorar o funcionamento do poço em eventos de chuva de maior volume total precipitado seria necessário revisar os parâmetros de cálculo estabelecidos na legislação do município de São Carlos com vistas a aumentar o volume total armazenado.

\section{CONCLUSÃO}

Tubulações devem ser dimensionadas considerando a intensidade máxima calculada em um período de retorno de projeto para correta condução da água, porém os dispositivos de controle da água da chuva podem ter seu dimensionamento ou funcionamento sustentado em outras metodologias. Os ECC são uma forma confiável de escolha de um evento pluviométrico histórico para simulação do comportamento da chuva em dispositivos de drenagem urbana compensatória. Os ECC nos dão ainda uma cronologia do evento de chuva mais precisa, mostrando as variações dos índices pluviométricos de acordo com a evolução temporal, que é particularmente útil no estudo de dispositivos de infiltração, retenção e evapotranspiração, permitindo uma melhor estimativa do balanço hídrico da solução.

É passível de questionamentos a forma de seleção dos percentis de chuva e consequentemente da quantidade de classes de eventos pluviométricos. Mudanças nos padrões adotados nesse trabalho irão modificar consideravelmente a escolha dos ECC. Obviamente esses intervalos podem ser ajustados de acordo com os interesses de cada pesquisa. Outro ponto de destaque é a escolha do período entre eventos, que neste trabalhou foi adotado de 24h. Períodos mais curtos levarão a determinação de uma maior quantidade de eventos.

Quanto a duração horária dos dados de chuva utilizados no trabalho, considera-se ser a máxima recomendada para determinação de eventos pluviométricos. A utilização de uma escala menor, como a cada 5 minutos, nos forneceria uma evolução do evento mais realista, porém além dos objetivos do estudo. Outrossim, deve ser considerado que a extração de eventos pluviométricos de uma série de dados históricos demanda recursos computacionais consideráveis. Registros pluviométricos com escala de poucos minutos, intervalos entre eventos muito curtos e séries muito longas podem levar ao esgotamento da capacidade de processamento de computadores regulares.

A simulação do comportamento de poços de infiltração quando submetidos aos eventos de chuva é simples e de grande utilidade para o planejamento urbano. Nestas simulações não foram consideradas condições antecedentes de umidade do solo, o que irá alterar significativamente a resposta real do poço. Para superar essa barreira seria necessário adotar técnicas de simulação contínua, que também demandam recursos computacionais significativos.

Deste modo, os ECC podem ser utilizados para a modelagem de diversos dispositivos de drenagem urbana sustentável. Além disso, o correto entendimento da cronologia da chuva pode refletir em custos menores, funcionamento hidráulico adequado e aumentar as opções de desenho arquitetônico e urbanístico. Por fim, salienta-se que o problema do escoamento superficial da água de chuva no meio urbano exige soluções multidisciplinares e os ECC podem ser um instrumento de apoio aos diversos profissionais da água.

\section{REFERÊNCIAS}

Alikhani, J., Nietch, C., Jacobs, S., Shuster, B., \& Massoudieh, A. (2019). Modeling and design scenario analysis of long-term monitored bioretention system for rainfall-runoff reduction to combined sewer in Cincinnati, $\mathrm{OH}$. Journal of Sustainable Water in the Built Environment, 6(2), 1-17.

Andersen, J. S., Lerer, S. M., Backhaus, A., Jensen, M. B., \& Sørup, H. J. D. (2017). Characteristic rain events: a methodology for improving the amenity value of stormwater control measures. Sustainability, 9(10), 1793. 
Ashley, R. M., Walker, A. L., D’Arcy, B., Wilson, S., Illman, S., Shaffer, P., Woods-Ballard, B., \& Chatfield, P. (2015) UK sustainable drainage systems: past, present and future. Proceedings of ICE - Civil Engineering, 168(3), 125-130.

Backhaus, A., \& Fryd, O. (2013). The aesthetic performance of urban landscape-based stormwater management systems: a review of twenty projects in Northern Europe. Journal of Landscape Architecture, 8(2), 52-63.

Ballard, B. W., Wilson, S., Udale-Clarke, H., Illman, S., Scott, T., Ashley, R., \& Kellagher, R. (2015). The SuDS manual. Londres: CIRIA.

Baptista, M., Nascimento, N., \& Barraud, S. (2011). Técnicas compensatórias em drenagem urbana (2. ed.). Porto Alegre: Associação Brasileira de Recursos Hídricos.

Beecham, S., \& Chowdhury, R. (2012). Effects of changing rainfall patterns on WSUD in Australia. Water Management, 165(5), 285-298.

Bemfica, D. D. C., Goldenfum, J. A., \& Silveira, A. L. L. D. (2000). Análise da aplicabilidade de padrões de chuva de projeto a Porto Alegre. Revista Brasileira de Recursos Hídricos, 5(4), 5-16.

Brasil. (2007). Águas pluviais: técnicas compensatórias para o controle de cheias urbanas: guia do profissional em treinamento nível 2/3 (52 p.). Belo Horizonte: Ministério das Cidades.

DiGiovanni, K. (2018). Sustainability. Water Environment Research, 90(10), 1596-1619. http://dx.doi.org/10.2175/106143018X15289915807326

Dunkerley, D. (2015). Intra-event intermittency of rainfall: an analysis of the metrics of rain and no-rain periods. Hydrological Processes, 29, 3294-3305.

Ferreira, T. S. (2016). Avaliação do comportamento hidrológico de poços de infiltração de águas pluviais sob diferentes concepções (157 p.). São Carlos.

Gaál, L., Molnar, P., \& Szolgay, J. (2014). Selection of intense rainfall events based on intensity thresholds and lightning data in Switzerland. Hydrology and Earth System Sciences, 18, 1561-1573.

Instituto Brasileiro de Geografia e Estatística - IBGE. (2001). Tendências demográficas (63p.). Rio de Janeiro.

Instituto Brasileiro de Geografia e Estatística - IBGE. (2018). Projeção populacional 2018. Rio de Janeiro.

Instituto Nacional de Meteorologia - INMET. (2011). Nota técnica: rede de estações meteorológicas automáticas do INMET (pp. 11, № 001/2011/ SEGER/LAIME/CSC/INMET).

Khan, S. T., Chapa, F., \& Hack, J. (2020). Highly resolved rainfall-runoff simulation of retrofitted green stormwater infrastructure at the micro-watershed scale. Land, 9(9), 339.

Marsalek, J., Jiménez-Cisneros, B., Karamouz, M., Malmquist, P., Goldenfum, J., \& Chocat, B. Urban water cycle processes. Leiden: Taylor \& Francis, v. 2, 2008.

Oliveira, A. P. D., Barbassa, A. P., \& Gonçalves, L. M. (2016). Aplicação de técnicas compensatórias de drenagem na requalificação de áreas verdes urbanas em guarulhos - sp. Cidades Verdes, 4(9), 87-101.

Palynchuk, B., \& Guo, Y. (2007). Threshold analysis of rainstorm depth and duration statistics at Toronto, Canada. Journal of Hydrology, 348, 535-545.

Peroni, C. S. L., \& Teixeira, B. A. D. N. (2019). Evaluation of rainwater detention basins in the urban area of a middle sized city in Brazil. NOVATECH, 1-4.

Rawls, W. J., Brakensiek, D. L., \& Miller, N. (1983). Green-ampt infiltration parameters from soils data. Journal of Hydraulic Engineering, 109(1), 62-70.

Silveira, A. L. L. D., \& Goldenfum, J. A. (2007). Metodologia generalizada para pré-dimensionamento de dispositivos de controle pluvial na fonte. Revista Brasileira de Recursos Hídricos, 12(2), 157-168.

Sørup, H. J. D., Lerer, S. M., Arnbjerg-Nielsen, K., Mikkelsen, P. S., \& Rygaard, M. (2016). Efficiency of stormwater control measures for combined sewer retrofitting under varying rain conditions: quantifying the Three Points Approach (3PA). Environmental Science \& Policy, 63, 19-26.

Tucci, C. E. M. (2003). Inundações e drenagem urbana. In C. E. M. Tucci \& J. C. Bertoni (Eds.), Inundações urbanas na América do Sul (Cap. 3, 1. ed.). Porto Alegre: Associação Brasileira de Recursos Hidrícos.

United Nations. (2018). World urbanization prospects: the 2018 revision. New York: Department of Economic and Social Affairs, Population Division. 


\section{Contribuições dos autores:}

Luan Serafim Mendes Gonçalves: concepção e planejamento do estudo; Obtenção, análise e interpretação dos dados; Redação do texto e padronização das normas de acordo com a revista.

Bernardo Arantes do Nascimento Teixeira: concepção e planejamento do estudo; Obtenção, análise e interpretação dos dados; Revisão do texto. 